ФТИ-2019

\title{
3D DENDRITE SHAPE IN THE LARGE CHEMICAL PÉCLET NUMBER LIMIT IN THE CASE OF ROTATIONAL SYMMETRY
}

\author{
Titova E.A. , Alexandrov D.V. \\ Ural Federal University, Yekaterinburg, Russia \\ *E-mail: ekatitova@mail.ru
}

The solution of nonlinear integrodifferential equation for the mass transport defines the shape of dendrite, growing in a supersaturated alloy. We obtained a solution of this equation in the limit of large chemical Péclet number in the case when dendrite shape is a surface of revolution.

The equation (1) describes the shape of the growing dendrite as a function of supersaturation in steady-state non-equilibrium conditions [1]:

$$
\begin{gathered}
\frac{Q}{m_{v} c_{p}}\left[\frac{d_{c}}{\rho} K+\beta V\right]-C_{l \infty}=I_{\zeta}^{C H} \\
I_{\zeta}^{C H}=\frac{2\left(1-k_{v}(V)\right)}{\sqrt{1-P_{C} \tau_{*}}}\left(\frac{P_{C}}{2 \pi}\right)^{3 / 2} \int_{-\infty}^{\infty} \int_{-\infty}^{\infty} K_{1 / 2}\left(\frac{P_{C} \sqrt{b_{H}}}{1-P_{C} \tau_{*}}\right) \frac{C_{i}\left(\mathbf{x}_{1}\right)}{b_{H}^{1 / 4}} \exp \left(-\frac{P_{C}\left(\zeta(\mathbf{x})-\zeta\left(\mathbf{x}_{1}\right)\right)}{1-P_{C} \tau_{*}}\right) d^{2} x_{1}
\end{gathered}
$$

where $\zeta$ is a moving phase transition interface, $V$ - constant velocity of growth, 2 1 terfacial concentration, $b_{H}=\left(1-P_{C} \tau_{*}\right)\left|\mathbf{x}-\mathbf{x}_{1}\right|^{2}+\left(\zeta(\mathbf{x})-\zeta\left(\mathbf{x}_{1}\right)\right)^{2}$ and $K$ is an interfacial curvature. Retaining in the Bessel function only the leading term we get the two-dimensional Laplase-type integral that can be calculated with a saddle-point technique:

$$
I_{\zeta}^{C H}=\frac{\left(1-k_{v}(V)\right) C_{i}\left(x_{0}, y_{0}\right)\left(1-P_{C} \tau_{*}\right) \exp \left(\frac{P_{C} S\left(x_{0}, y_{0}\right)}{1-P_{C} \tau_{*}}\right)}{\sqrt{\left(\zeta_{x_{1} x_{1}}^{\prime \prime} S\left(x_{0}, y_{0}\right)+\left(1-P_{C} \tau_{*}\right)\right)\left(\zeta_{y_{1} y_{1}}^{\prime \prime} S\left(x_{0}, y_{0}\right)+\left(1-P_{C} \tau_{*}\right)\right)-\left(\zeta_{x_{1} y_{1}}^{\prime \prime} S\left(x_{0}, y_{0}\right)\right)^{2}}}
$$

where $S\left(x_{1}, y_{1}\right)=\sqrt{b_{H}}+\zeta(x, y)-\zeta\left(x_{1}, y_{1}\right)$ and $x_{0}$ is a maximum point of $S$. Under assumption that interface function is a surface of revolution, we can consider only the section $y=0$. Then eq. (3) coincides with previously obtained equation for the 2D shape [2]. Expand the function $\zeta$ in a Taylor series in a vicinity of $x_{0}$ we realized that the integral contribution $I_{\zeta}^{C H}$ vanishes. 
Asymptotically obtained interfacial function $\zeta$ is a sphere. The numerical solution of equation (3) is also close to a sphere, as shown in Figure 1. This result is in a agreement with limiting case of the steady-state solidification conditions with high Péclet numbers [1].

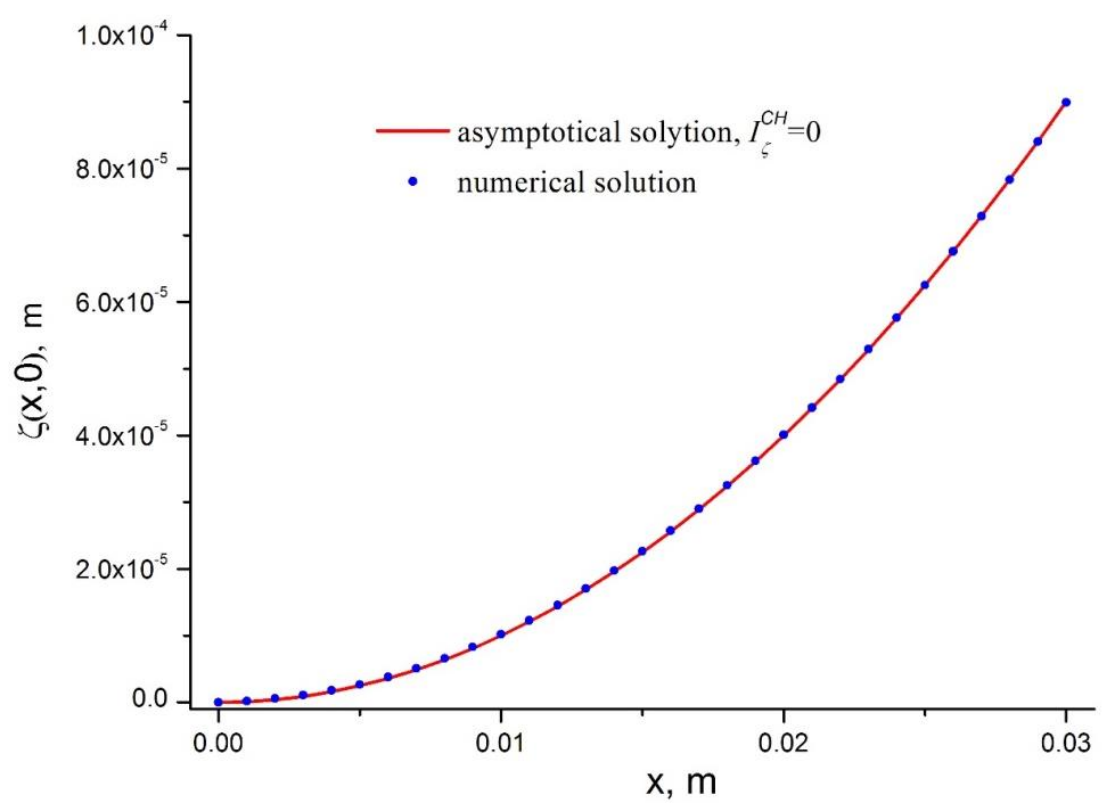

This work is supported by the Russian Science Foundation (grant no. 16-1110095).

1. Galenko P.K, Alexandrov D.V. and Titova E.A., Phil. Trans. R. Soc. A, (2017).

2. 2.Titova E. A. AIP Conference Proceedings 2015, 020102 (2018); doi: $10.1063 / 1.5055175$ 